\title{
Esfingídeos (Lepidoptera, Sphingidae) no Tabuleiro Paraibano, nordeste do Brasil: abundância, riqueza e relação com plantas esfingófilas
}

\author{
Reisla Oliveira Darrault ${ }^{1}$ \\ Clemens Schlindwein ${ }^{2}$
}

\begin{abstract}
Hawkmoths (Lepidoptera, Sphingidae) in the Tabuleiro Paraibano, northeastern Brazil: abundance, richness and relations to sphingophilous plants. Hawkmoths (Sphingidae) are among the major pollinators in tropical communities. Here the first survey of sphingids and related plants in Northeastern Brazil is presented. The sphingids were surveyed from March 1999 through April 2000 at the Reserva Biológica Guaribas, Mamanguape, Paraíba State. On black and mix light traps, 136 hawkmoths from 24 species were captured. Individuals of Erinnyis ello (Linnaeus, 1758), Isognathus menechus (Boisduval, 1875) and Xylophanes tersa (Linnaeus, 1771) represented $58 \%$ of the samples. One half of the recorded species show wide distribution in the Neotropics. Three species were registered for more than six months. Most of the species were found only in the dry or wet season. Pollen from 34 plant species were recorded by pollen analysis of sphingid mouth parts. Hancornia speciosa (Apocynaceae) and Guettarda platipoda (Rubiaceae) were the most important food plants. Both are characteristic elements of the Tabuleiro Nordestino and present typically sphingophilous flowers. More than one half of the sphingids presented pollen from only one or two species of plants. By transporting large quantities of pollen of a few species, hawkmoths seem to be the main pollinators of nocturnal flowers in the Tabuleiro Paraibano.

KEY WORDS. Sphingidae, Tabuleiro Nordestino, hawkmoths, biodiversity, sphingophilous plants, pollen analysis, Northeastern Brazil
\end{abstract}

Com aproximadamente 1050 espécies, os esfingídeos (Sphingidae) compõem um grupo largamente distribuído, ocorrente em todos os continentes (D'ABRERA 1986; KITCHING \& CADIOU 2000). A maior parte das espécies conhecidas (75\%) é encontrada nas regiões tropicais. A América do Sul possui pelo menos 260 espécies (JANZEN 1983).

De um modo geral, os esfingídeos são bem representados em coleções entomológicas de todo o mundo (KITCHING \& CADIOU 2000). Apesar disso, há pouca informação sobre a composição e a distribuição da esfingofauna brasileira.

Todos os levantamentos realizados no Brasil ocorreram nas regiões Sul e Sudeste do país, em áreas de Mata Atlântica (FERREIRA et al. 1986), mata subtropical (LAROCA \& MIELKE 1975; LAROCA et al. 1989; MARINONI et al. 1999), em campos (BIEZANKO 1948), Mata de Araucária e Mata Tropical Úmida (MARINONI et al. 1999).

1) Departamento de Sistemática e Ecologia, Universidade Federal da Paraíba. Cidade Universitária, 58059-900 João Pessoa, Paraíba, Brasil.

2) Correspondências: Departamento de Botânica, Universidade Federal de Pernambuco. Avenida Prof. Moraes Rego, Cidade Universitária, 50670-901 Recife, Pernambuco, Brasil. 
A esfingofauna do Nordeste brasileiro ainda não foi estudada sistematicamente. Nesta região foram documentados apenas registros isolados da ocorrência de esfingídeos (veja em D’ABRERA 1986; LOCATELLI \& MACHADO 1999).

O ciclo de vida dos esfingídeos é bastante uniforme. Os ovos são depositados em folhas de plantas hospedeiras. Após a eclosão as larvas se alimentam por três a quatro semanas e deixam as plantas hospedeiras em busca de um local para se empupar. Em geral, as lagartas são altamente especializadas em relação às plantas hospedeiras que utilizam. Indivíduos de uma população alimentam-se de uma ou poucas espécies de plantas. Populações da mesma espécie e ambientes diferentes geralmente utilizam plantas hospedeiras aparentadas (JANZEN 1983).

Os adultos alimentam-se de néctar e são polinizadores efetivos, sobretudo em comunidades tropicais (SILBERBAUER-GOTTSBERGER \& GOTTSBERGER 1975; HABER \& FRANKIE 1989; BAWA 1990), onde são adaptados às flores de espécies arbóreas, arbustivas e trepadeiras (HABER \& FRANKIE 1982). São forrageadores predominantemente noturnos e polinizam tipicamente plantas esfingófilas, com flores brancas, tubulares, hipocrateriformes ou em pincel e com um forte aroma à noite (VOGEL 1954; FAEGRI \& VAN DER PIJL 1979, HABER \& FRANKIE 1989).

Em ambientes tropicais com estações secas e chuvosas claramente delimitadas, a forte sazonalidade influencia a história de vida dos animais e plantas que habitam estes locais (e.g. JANZEN 1967; FRANKIE et al. 1974; WOLDA 1978; JANZEN 1983; HABER \& FRANKIE 1989). Por dependerem das plantas como fontes de recursos alimentares para larvas (herbívoras) e adultos (nectarívoros), a ocorrência e a abundância dos esfingídeos também são influenciadas pela variação sazonal da disponibilidade do recurso alimentar ocorrente naqueles ambientes.

Neste estudo apresenta-se o primeiro levantamento de esfingídeos no Nordeste do Brasil, ocorrentes numa área do Tabuleiro da Paraibano, sua sazonalidade e relações com plantas esfingófilas.

\section{MATERIAL E MÉTODOS}

\section{Área de estudo}

O estudo foi conduzido em uma área da Reserva Biológica Guaribas (IBAMA) (06 44'32”'S; 3508'33'W), no município de Mamanguape, litoral norte da Paraíba.

A vegetação da área de coleta é uma savana arbórea aberta (PRATES et al. 1981), conhecida como tabuleiro (OliveIRA-FilHO \& CARVALHO 1993). Tal formação é considerada uma ocorrência disjunta do cerrado em áreas de solos arenosos, próximas ao litoral, sobre a Formação Geológica Barreiras (ANDRADE-LIMA 1960). A vegetação do tabuleiro é caracterizada por árvores e arbustos esparsos ou agrupados em manchas e por uma cobertura herbácea. Entre os arbustos e árvores predominam espécies típicas dos cerrados do Brasil Central (Oliveira-FILHo et al. 1986), como Hancornia speciosa (Apocynaceae), Curatela americana (Dilleniaceae), Byrsonima cydoniifolia (Malpighiaceae) e Bowdichia virgilioides (Fabaceae), além de espécies peculiares do Tabuleiro Nordestino, como Campomanesia dicotoma (Myrtaceae), Guettarda platipoda (Rubiaceae), Hirtella ciliata, H. racemosa (Chrysobalanaceae) e Ouratea fieldingiana (Ochnaceae) (ANDRADE-LIMA 1960; TAVARES 1988a,b). 
Na Reserva Biológica Guaribas, as estações seca e chuvosa são marcantes de forma que precipitações máximas ocorrem de março a junho e uma estiagem de setembro a dezembro (Fig. 1). As temperaturas médias mensais oscilam entre 24-26으 (IBAMA 1995).
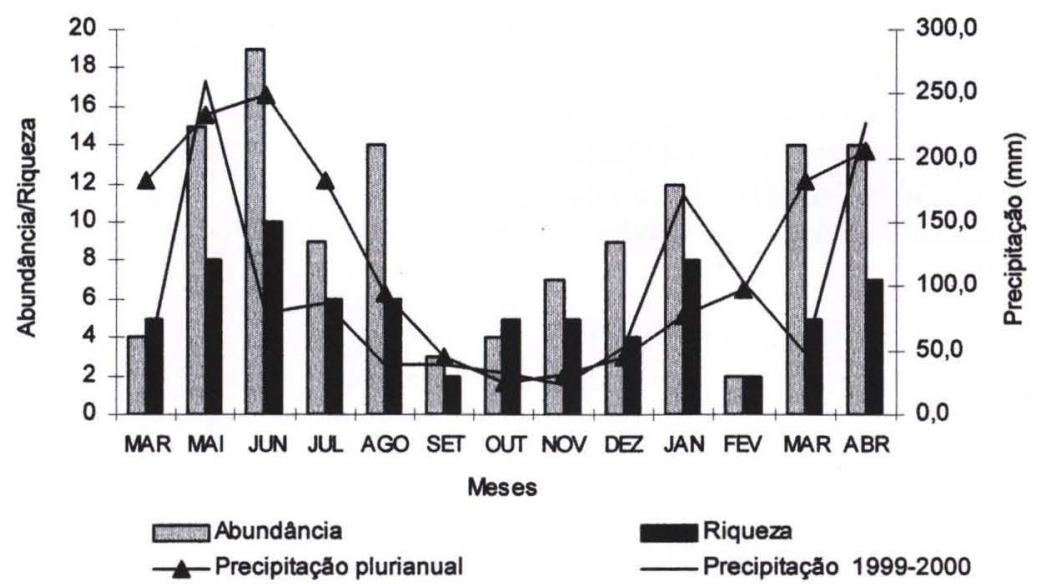

Fig. 1. Variação sazonal da riqueza de espécies e da abundância de esfingídeos (março 1999 a abril 2000) e valores de precipitação média mensal plurianual (1910 a 1985) e do período de coleta (1999-2000) na Reserva Biológica Guaribas.

\section{Coleta dos esfingídeos}

As coletas dos esfingídeos foram realizadas uma vez por mês de março de 1999 a abril de 2000. Os esfingídeos foram atraídos por uma lâmpada de luz negra instalada em meio à vegetação de tabuleiro, à frente de um lençol branco estendido verticalmente entre duas pilastras de madeira. Também foram coletados esfingídeos atraídos por cinco lâmpadas de luz mista utilizadas para a iluminação da sede da reserva.

As coletas foram realizadas em noites de lua nova, já que a luz lunar diminui a atratividade dos esfingídeos pela luz artificial (LAROCA \& MIELKE 1975; JANZEN 1983; STEINER \& NIKUSH 1994).

Os esfingídeos foram coletados com uso de uma rede entomológica ou manualmente, do crepúsculo (17:30-18:00 h) ao amanhecer (05:00-06:00 h). Indivíduos observados fora do horário de coleta ou ao visitarem flores também foram capturados. Estas coletas forneceram informações adicionais sobre sazonalidade e composição em espécies de esfingídeos e sobre suas fontes de alimento.

Os espécimes coletados foram montados, etiquetados e depositados na coleção entomológica do Departamento de Sistemática e Ecologia da Universidade Federal da Paraíba (UFPB) e na do Departamento de Botânica da Universidade Federal de Pernambuco (UFPE).

Os esfingídeos foram identificados em cooperação com o Dr. Olaf Mielke da Universidade Federal do Paraná (UFPR) e com o auxílio de pranchas e descrições 
de D'ABRERA (1986). A nomenclatura utilizada foi atualizada de acordo com KITCHING \& CADIOU (2000).

Dados de pluviosidades médias mensais foram obtidos do posto pluviométrico de Mamanguape, do Laboratório de Meteorologia, Recursos Hídricos e Sensoriamento Remoto da Paraíba. Os valores de temperaturas mínima e máxima foram fornecidos pelo Centro de Ciências e Tecnologia do Departamento de Ciências Atmosféricas da UFPB.

Para se verificar a relação entre as variações das características climáticas e a riqueza de espécies e abundância dos esfingídeos capturados, foi feita uma correlação linear simples entre o número de espécies e de indivíduos coletados e cada variável climática. As correlações foram realizadas pelo programa SPSS 7.5 for Windows.

\section{Relação com plantas esfingófilas}

As espirotrombas dos indivíduos coletados foram analisadas em estereo-microscópio quanto à presença de grãos de pólen. Enquanto eram mantidas esticadas, as espirotrombas dos esfingídeos eram pressionadas com pequenas porções de gelatina glicerinada corada com fucsina básica onde os grãos de pólen ficavam aderidos. A gelatina com os grãos era transferida para lâminas aquecidas em lamparinas com álcool, cobertas com uma lamínula e seladas com parafina (LOUVEAUX et al. 1978; WITTMANN \& SCHLINDWEIN 1995).

Os grãos de pólen foram identificados com uso de um microscópio (Zeiss Standart 25), por comparação com lâminas da palinoteca do Laboratório de Ecologia Vegetal da UFPE. Fotos em microscopia eletrônica de varredura foram feitas com um aparelho Zeiss DSM 940.

Foram montadas exsicatas e lâminas de pólen de plantas esfingófilas da área de estudo. As exsicatas foram depositadas no Herbário Lauro Pires Xavier do Departamento de Sistemática e Ecologia da UFPB e no Herbário Geraldo Mariz (UPE) do Departamento de Botânica da UFPE. As lâminas foram depositadas na palinoteca do Laboratório de Ecologia Vegetal (UFPE).

\section{RESULTADOS}

\section{Riqueza, composição em espécies, abundância e sazonalidade}

Foram coletadas 136 mariposas de 24 espécies (Tab. I). Indivíduos de Erinnyis ello, Isognathus menechus (Boisduval, 1875) e Xylophanes tersa (Linnaeus, 1771) representaram $58 \%$ da amostra. Sete espécies (29\%) foram representadas por apenas um indivíduo.

De um modo geral, foi verificada uma maior riqueza de espécies nos meses de maior abundância de esfingídeos ( $r=0,859$; Fig. 1).

Sete espécies foram amostradas apenas nos meses chuvosos (março a julho) e oito apenas nos meses secos (agosto a fevereiro). As demais (9) foram registradas em meses secos e chuvosos. Junho foi o mês de maior abundância e o de maior riqueza. Em fevereiro, foram coletados apenas um indivíduo de Enyo ocypete e um de Perigonia pallida (Tab. II). 
Tabela I. Abundância das espécies de esfingídeos registradas na Reserva Biológica Guaribas de março de 1999 a abril de 2000.

\begin{tabular}{|c|c|c|c|}
\hline Espécies & Luz mista & Luz negra & Total \\
\hline \multicolumn{4}{|l|}{ Macroglossinae } \\
\hline \multicolumn{4}{|l|}{ Macroglossini } \\
\hline Hyles euphorbiarum (Guérin-Méneville \& Percheron, 1835 & 1 & 1 & 2 \\
\hline Xylophanes tersa (Linnaeus, 1771) & 10 & 2 & 12 \\
\hline \multicolumn{4}{|l|}{ Dilophonotini } \\
\hline Aellopos fadus (Cramer, 1777) & 2 & 0 & 1 \\
\hline Aellopos titan (Cramer, 1777) & 0 & 1 & 1 \\
\hline Callionima grisescens (Rothschild, 1894) & 0 & 3 & 3 \\
\hline Callionima parce (Fabricius, 1775) & 1 & 1 & 2 \\
\hline Enyo ocypete (Linnaeus, 1758) & 3 & 2 & 5 \\
\hline Erinnyis crameri (Schaus, 1898) & 2 & 0 & 2 \\
\hline Erinnyis ello (Linnaeus, 1758) & 25 & 6 & 31 \\
\hline Erinnyis oenotrus (Cramer, 1780) & 1 & 0 & 1 \\
\hline Isognathus caricae (Linnaeus, 1758) & 5 & 1 & 6 \\
\hline Isognathus menechus (Boisduval, [1875]) & 30 & 4 & 34 \\
\hline Madoryx oiclus (Cramer, 1779) & 1 & 0 & 1 \\
\hline Pachylia syces (Hübner, [1819]) & 1 & 0 & 1 \\
\hline Pachylia ficus Linnaeus, 1758 & 0 & 1 & 1 \\
\hline Perigonia pallida Rothschild \& Jordan, 1903 & 1 & 2 & 3 \\
\hline Pseudosphinx tetrio (Linnaeus, 1771) & 4 & 0 & 4 \\
\hline \multicolumn{4}{|l|}{ Smerinthinae } \\
\hline \multicolumn{4}{|l|}{ Ambulycini } \\
\hline Adhemarius palmeri (Boisduval, [1875]) & 6 & 1 & 7 \\
\hline Protambulyx strigilis (Linnaeus, 1771) & 1 & 0 & 1 \\
\hline \multicolumn{4}{|l|}{ Sphinginae } \\
\hline \multicolumn{4}{|l|}{ Acherontiini } \\
\hline Agrius cingulatus (Fabricius, 1775) & 7 & 2 & 9 \\
\hline \multicolumn{4}{|l|}{ Sphingini } \\
\hline Cocytius antaeus (Drury, 1773) & 1 & 3 & 4 \\
\hline Manduca diffissa (Butler, 1871) & 1 & 0 & 1 \\
\hline Manduca sexta paphus (Cramer, 1779) & 1 & 0 & 1 \\
\hline Neogene dynaeus (Hübner, [1827]-[1831]) & 0 & 3 & 3 \\
\hline Total & 103 & 33 & 136 \\
\hline
\end{tabular}

Das 24 espécies amostradas, 14 (58\%) ocorreram em apenas um ou dois meses do ano. Por outro lado, espécimes de Erinnyis ello (Linnaeus, 1758) e Isognathus menechus foram registrados em pelo menos 10 dos 13 meses de coleta. Indivíduos de Xylophanes tersa foram coletados em todos os meses do ano, exceto em janeiro e fevereiro, os dois últimos meses da estação seca (Tab. II).

A abundância e a riqueza dos esfingídeos se correlacionaram de forma significativamente positiva com a pluviosidade plurianual. Contudo, nenhuma das demais variáveis ambientais medidas, inclusive a pluviosidade ocorrida no período de coleta, apresentou correlação significativa com a abundância e a riqueza dos esfingídeos (Tab. III).

\section{Relação com plantas esfingófilas}

Através da análise polínica da espirotromba dos esfingídeos foram registradas 34 espécies de plantas (Tab. IV, Fig. 2). Nove tipos polínicos não foram identificados até o nível de família. Dentre as plantas visitadas pelos esfingídeos, ocorreram arbustos, árvores e trepadeiras. 
Tabela II. Distribuição sazonal dos esfingídeos coletados em luzes negra e mista na Reserva Biológica Guaribas (março 1999 a abril 2000). (A) Número de tipos polínicos registrados nas espirotrombas e (B) comprimento médio das espirotrombas dos esfingídeos.

\begin{tabular}{|c|c|c|c|c|c|c|c|c|c|c|c|c|c|c|c|}
\hline \multirow{2}{*}{ Espécies } & \multicolumn{13}{|c|}{ Meses } & \multirow{2}{*}{ A } & \multirow{2}{*}{$\begin{array}{c}\mathrm{B}(\mathrm{cm}) \\
\text { (amplitude total; } \mathrm{n})\end{array}$} \\
\hline & Mar & Mai & Jun & Jul & Ago & Set & Out & Nov & Dez & Jan & Fev & Mar & $\mathrm{Abr}$ & & \\
\hline Adhemarius palmeri & & - & - & - & & & & & & - & & & & 5 & $2,4(0,3 ; 4)$ \\
\hline Aellopos fadus & & & & & & & & & & - & & & & 4 & $1,4(1)$ \\
\hline Aellopos titan & - & & & & & & & & & & & & & 0 & - \\
\hline Agrius cingulatus & - & & - & & - & - & & & & & & & - & 11 & $9,0(0,8 ; 6)$ \\
\hline Callionima grisescens & & & & & - & & & & & & & & & 1 & $1,7(1)$ \\
\hline Callionima parce & & & & - & - & & & & & & & & & 0 & $2,2(1,0,1 ; 2)$ \\
\hline Cocytius antaeus & & & - & & & & & & & & & - & - & 3 & $10,2(3,1 ; 3)$ \\
\hline Enyo ocypete & & & & - & & & & & - & - & - & & - & 7 & $2,2(3)$ \\
\hline Erinnyis crameri & & - & & & & & & & & & & - & & 4 & $3,5(10,7 ; 2)$ \\
\hline Erinnyis ello & - & - & - & & & & - & & - & - & & - & - & 22 & $3,4(0,3 ; 21)$ \\
\hline Erinnyis oenotrus & - & & & & & & & & & & & & & 3 & $3,7(1)$ \\
\hline Hyles euphorbiarum & & & & & & & & & - & - & & & & 8 & $2,5(1)$ \\
\hline Isognathus caricae & & - & & - & & & - & - & & & & & & 7 & $4,0(0,4 ; 3)$ \\
\hline Isognathus menechus & & - & - & - & - & & - & - & - & - & & - & - & 23 & $3,9(0,9 ; 22)$ \\
\hline Madoryx oiclus & & & - & & & & & & & & & & & 0 & - \\
\hline Manduca diffissa & & & & & & & & - & & & & & & 2 & $8,2(1)$ \\
\hline Manduca sexta paphus & & & & & & & & & & - & & & & 3 & $7,3(1,7 ; 3)$ \\
\hline Neogene dynaeus & & & & & & & & & & - & & & & 4 & $1,5(0,2 ; 2)$ \\
\hline Pachylia ficus & & & & & - & & & & & & & & & 1 & $3,7(1)$ \\
\hline Pachylia syces & & & & & & & - & & & & & & & 0 & - \\
\hline Perigonia pallida & & - & - & & & & & & & & - & & & 3 & $1,4(1)$ \\
\hline Protambulyx strigilis & & & & & - & & & & & & & & & 1 & $2,7(1)$ \\
\hline Pseudosphinx tetrio & & - & - & & & & & & & & & & & 5 & $5,8(2,0 ; 2)$ \\
\hline Xylophanes tersa & - & - & - & - & - & - & - & - & - & & & - & - & 10 & $3,4(0,8 ; 9)$ \\
\hline
\end{tabular}

Observações: março a julho = estação chuvosa; agosto a fevereiro = estação seca; (-) ocorrência de indivíduos.

Tabela III. Coeficientes de correlação linear de Pearson ( $r$ ) entre a riqueza e a abundância de esfingídeos e variáveis climáticas medidas na Reserva Biológica Guaribas.

\begin{tabular}{lcc}
\hline & \multicolumn{2}{c}{ Coeficientes de correlação linear de Pearson ( $r)$} \\
\cline { 2 - 3 } & Riqueza & Abundância \\
\hline Pluviosidade média mensal plurianual (1910 a 1985) & $0,643^{*}$ & $0,610^{*}$ \\
Pluviosidade média mensal (1999-2000) & 0,473 & 0,446 \\
Temperatura mínima (1999-2000) & $-0,033$ & - \\
Temperatura máxima (1999-2000) & $-0,359$ & - \\
\hline
\end{tabular}

*) r significativo ao nível de significância $<0,05$.

O comprimento das espirotrombas dos esfingídeos variou de $1,4 \mathrm{~cm}$ (Aellopos fadus) a 10,5 cm (Cocytius antaeus) (Tab. II). O comprimento médio foi 3,6 $\mathrm{cm}$, e o mediano $3,1 \mathrm{~cm}$. Esfingídeos grandes, como Cocytius antaeus, Agrius cingulatus e Manduca sexta paphus exibiram as espirotrombas mais longas.

Houve uma grande variação na quantidade de pólen encontrado nas espirotrombas dos esfingídeos. O número de grãos de pólen registrados por indivíduo variou de 0 a 2,776. Não houve relação entre o comprimento da espirotromba e o número de grãos coletados (Fig. 3).

O número de tipos polínicos registrados nas peças bucais de um indivíduo variou de 1 a 6 (Fig. 4). Em 2/3 dos esfingídeos foi encontrado pólen de uma a duas espécies de plantas (1/3 das mariposas em cada caso). Cerca de $27 \%$ dos indivíduos apresentaram mais de três tipos polínicos nas espirotrombas. 

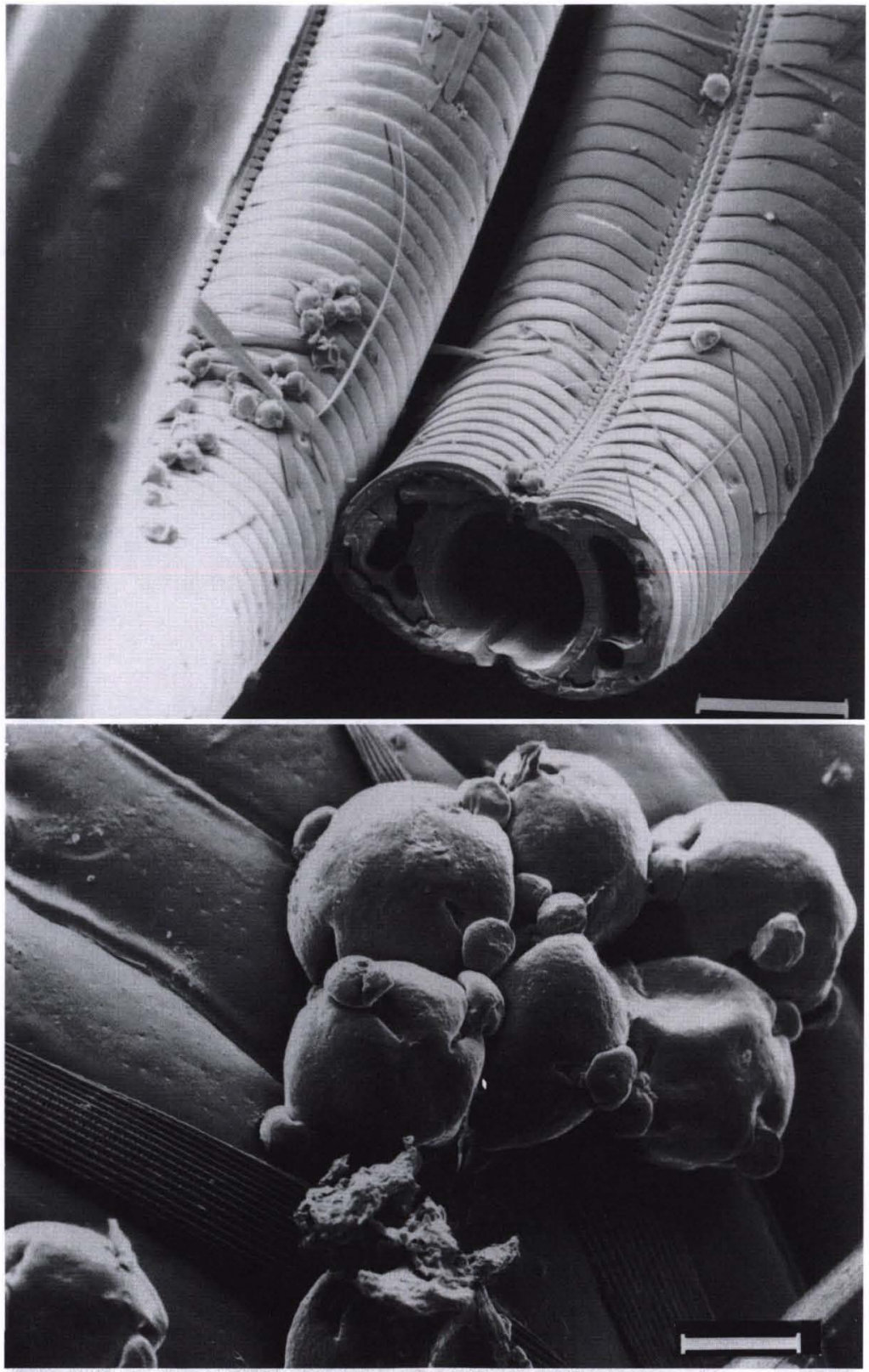

Fig. 2. Espirotromba de /sognathus menechus, microscopia eletrônica de varredura. a- seção, espirotromba enrolada, grãos de pólen aderidos à superfície da espirotromba, Escala $=200$ $\mu \mathrm{m}$; (b) pólen de Hancornia speciosa aderido à face ventral da espirotromba. Escala $=20 \mu \mathrm{m}$. 
Tabela IV. Plantas visitadas por esfingídeos em áreas de tabuleiro na Reserva Biológica Guaribas. Os dados são provenientes da análise polínica das espirotrombas dos esfingídeos. Cada tipo polínico representa um espécie de planta.

\begin{tabular}{|c|c|c|c|c|c|c|c|c|c|c|c|c|c|}
\hline \multirow{2}{*}{ Plantas } & \multicolumn{12}{|c|}{ Meses em que recebeu visitas } & \multirow{2}{*}{ Forma da flor } \\
\hline & Mar & $\mathrm{Abr}$ & Mai & Jun & Jul & Ago & Set & Out & Nov & Dez & Jan & Fev & \\
\hline \multicolumn{14}{|l|}{ Apocynaceae } \\
\hline Hancornia speciosa (Gomes) & & & & & & & & + & + & + & + & & Hipocrateriforme \\
\hline Mandevilla scabra * & & & & & & & & & & & & & Goela/infundibuliforme \\
\hline \multicolumn{14}{|l|}{ Asteraceae } \\
\hline sp. 1 & + & + & & + & + & + & & & + & + & + & & \\
\hline \multicolumn{14}{|l|}{ Bignoniaceae } \\
\hline Pithecoctenium sp. 1 & + & + & & & & & + & & & & & & Goela \\
\hline 3-zono-colporado & & & & & + & & & & & & & & \\
\hline \multicolumn{14}{|l|}{ Boraginaceae } \\
\hline Cordia sp. & + & + & & + & & + & & & & & & & Infundibuliforme \\
\hline Tournefortia sp. & & & & + & & & & & & & & & Infundibuliforme \\
\hline \multicolumn{14}{|l|}{ Capparidaceae } \\
\hline Capparis sp. & & & & + & & & + & & + & + & + & & Pincel \\
\hline \multicolumn{14}{|l|}{ Convolvulaceae } \\
\hline Ipomoea sp. & + & + & & & & & + & & & & & & Infundibuliforme \\
\hline 3-zono-colporado & & & & & + & & & & & + & & & \\
\hline \multicolumn{14}{|l|}{ Euphorbiaceae } \\
\hline Cnidoscolus sp. & & + & & & & & + & & + & + & & & Hipocrateriforme \\
\hline \multicolumn{14}{|l|}{ Loranthaceae } \\
\hline 3-zono-colporado & & & & & & & & & & & & + & \\
\hline \multicolumn{14}{|l|}{ Lythraceae } \\
\hline Cuphea flava Spreng. & & & + & & & & + & + & + & + & + & & Goela/ labiada \\
\hline \multicolumn{14}{|l|}{ Myrtaceae } \\
\hline 3-zono-colporado & & & & + & + & & & & & & & & Pincel/ disco \\
\hline 3-zono-colporado & & + & & & + & & & & + & & & & Pincel/ disco \\
\hline 3-zono-colporado & & & & + & & & + & & & & & & Pincel/ disco \\
\hline 3-zono-colporado & & & & & & & & & & + & & & Pincel/ disco \\
\hline \multicolumn{14}{|l|}{ Mimosaceae } \\
\hline Calliandra sp. & & & & & & & + & & & & + & & Pincel \\
\hline Inga sp.1 & & & & & & & & + & & + & + & & Pincel \\
\hline Inga sp.2 & & & & & & & & & & + & & & Pincel \\
\hline \multicolumn{14}{|l|}{ Rubiaceae } \\
\hline Guettarda platipoda & + & + & & + & + & + & & & + & & + & + & Hipocrateriforme \\
\hline Tocoyena sp." & & & & & & & & & & & & & Hipocrateriforme \\
\hline 8-zono-colporado & & & & + & & & + & + & & & + & & - \\
\hline zono-colporado & & & & & & & + & & & & & & - \\
\hline 8-zono-colporado & & & & + & & + & & & + & & & & - \\
\hline \multicolumn{14}{|l|}{ Sapindaceae } \\
\hline Serjania sp. & & & & & & & & & & & + & & Goela/labiada \\
\hline Tiliaceae & & & & & & & & & & & & & \\
\hline Luehea paniculata Mart. & & & & + & & & + & + & & + & & & Disco/ pincel \\
\hline Não identificados & & & & & & & & & & & & & \\
\hline 3-zono-colporado & + & & & + & & + & + & & + & & & & - \\
\hline zono-colporado & + & & & + & & & & & & & & + & - \\
\hline panto-porado & + & & & & & & & & & & & & - \\
\hline 3-panto-colporado & + & & & + & & & + & & + & & + & & - \\
\hline 4-zono-porado & + & + & & & & & + & & & & & & - \\
\hline 3-zono-colporado & & & & + & & & & & & & & & - \\
\hline zono-colporado & + & + & & & & & & & & + & & & - \\
\hline zono-colporado & & + & & & & & & & & + & & & - \\
\hline 3-zono-colporado & + & + & & + & & & & & & & + & & - \\
\hline
\end{tabular}

(*) Espécies de plantas esfingófilas, ocorrentes na área de estudo, cujo pólen não foi registrado nas peças bucais dos esfingídeos; (-) forma da flor não reconhecida; (+) Presença de visita. 


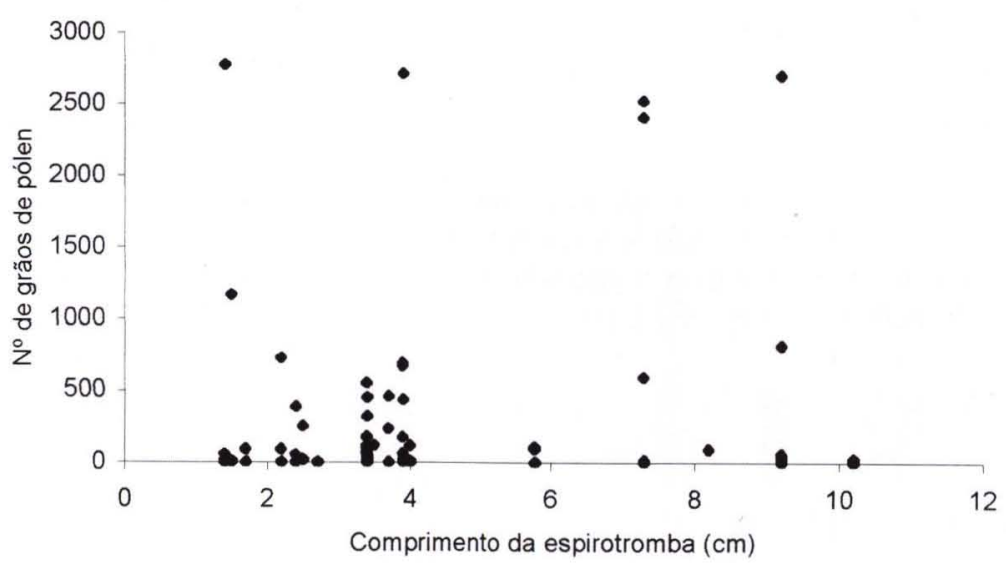

Fig. 3. Relação entre o comprimento das espirotrombas de esfingídeos e o número de grãos de pólen aderidos a elas. Mariposas com probóscides curtas ou longas apresentam quantidade semelhantes de pólen.

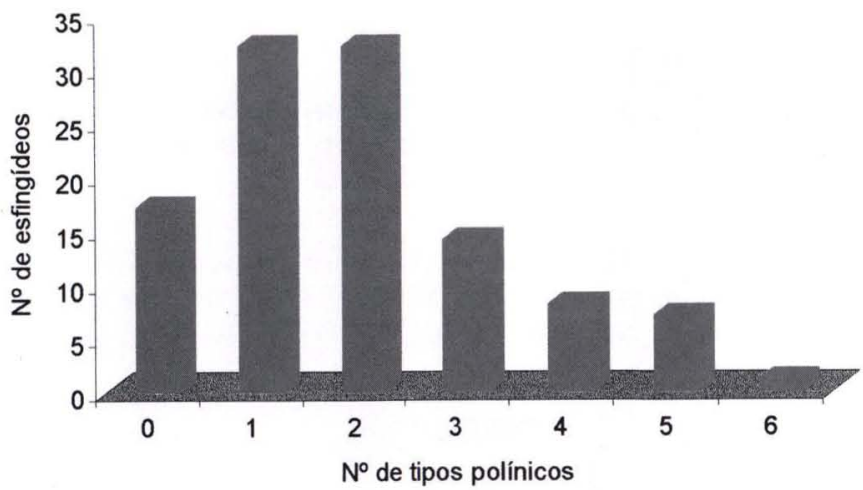

Fig. 4. Número de tipos polínicos registrados nas espirotrombas de esfingídeos. Na maioria dos indivíduos foi verificado pólen de 1 a 2 espécies de plantas.

O número de tipos polínicos registrados por mês na espirotromba dos esfingídeos variou de três (em fevereiro) a 15 (junho). Esse número não variou significativamente com os valores de precipitação $(r=0,139 ; p>0,05)$. Através dos grãos de pólen no aparelho bucal dos esfingídeos, foram registradas 26 espécies de plantas na estação chuvosa e 29 na seca. A maior parte das espécies vegetais (70\%) foi registrada nas duas estações. O número de tipos polínicos encontrados nas espirotrombas não variou de modo significativo com oscilações no número de indivíduos ou de espécies de esfingídeos ( $\mathrm{r}=0,14$ e 0,04, respectivamente).

O número de espécies de plantas utilizadas por espécie de esfingídeo variou de 1 a 23 (Fig. 5). Apenas três espécies de esfingídeos continham grãos de pólen de uma só espécie de planta. Em duas espécies, nenhum grão de pólen foi registrado. 
Isognathus menechus $\mathrm{e} X y$ lophanes tersa foram as espécies com a maior diversidade de plantas visitadas, apresentando 23 e 22 tipos polínicos nas peças bucais, respectivamente.

Guettarda platipoda e Hancornia speciosa foram visitadas, respectivamente, por $58 \%$ e $46 \%$ das espécies de esfingídeos amostradas. Cerca de $35 \%$ dos indivíduos amostrados visitaram as flores destas plantas. O tipo polínico de uma Asteraceae foi o terceiro mais registrado nas espirotrombas dos esfingídeos, ocorrendo em mais de $30 \%$ das espécies.

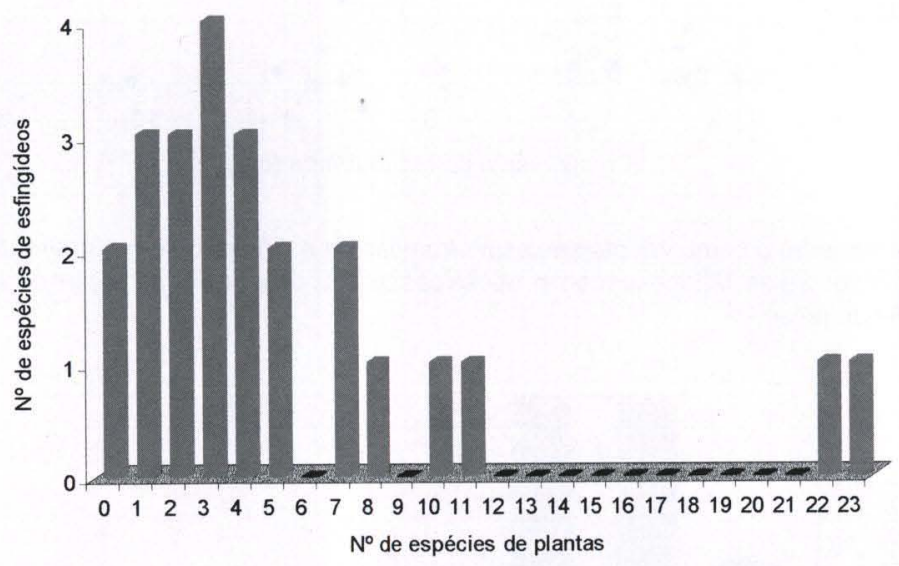

Fig. 5. Distribuição do número de espécies de plantas utilizadas por espécies de esfingídeos (dados obtidos através da análise de grãos de pólen encontrados nas espirotrombas dos esfingídeos). A maioria das espécies de esfingídeos exibe relação com mais de uma espécie de planta.

\section{DISCUSSÃO}

\section{Riqueza, abundância e sazonalidade dos esfingídeos}

Neste levantamento foram registrados cerca de $10 \%$ do total de esfingídeos estimados para a América do Sul (JANZEN 1983). Metade das espécies registradas na Reserva Biológica Guaribas é de vasta distribuição na região neotropical. Erinnyis ello, por exemplo, também foi uma das espécies dominantes em levantamentos no Paraná (LAROCA \& MIELKE 1975; MARINONI et al. 1999) e na Costa Rica (STEIFERT 1974). De acordo com D’ABRERA (1986), essa espécie é, potencialmente, a mais comum do Novo Mundo. As demais espécies amostradas são de ocorrência restrita à América do Sul (D’ABRERA 1986). Não foram registrados casos de endemismo.

A esfingofauna amostrada apresenta vários componentes comuns à fauna de esfingídeos registrada em levantamentos de outras regiões da América do Sul (Tab. V). A falta de padronização nas amostragens, sobretudo em relação ao número e tipo de lâmpadas e ao esforço amostral interfere na comparação dos resultados dos 
levantamentos. No entanto, parece que locais de clima e vegetação mais similares, mesmo que distantes, apresentam comunidades de esfingídeos de composições semelhantes. A esfingofauna registrada no tabuleiro paraibano, por exemplo, apresenta composição em espécies mais semelhante à de áreas de mata tropical seca na Costa Rica em relação à fauna de esfingídeos registrada em áreas com clima sub-tropical no Paraná (Tab. V).

Tabela V. Proporção relativa de espécies de esfingídeos registradas no Tabuleiro Paraibano e em outros tipos de vegetação. Todos os estudo realizados no Brasil ocorreram no Paraná.

\begin{tabular}{|c|c|c|c|c|c|c|}
\hline \multirow{2}{*}{ Tipo de vegetação } & \multirow{2}{*}{ Local } & \multirow{2}{*}{ Coletas/mês } & \multirow{2}{*}{$\begin{array}{c}\text { Total de } \\
\text { horas/mês * }\end{array}$} & \multicolumn{2}{|c|}{ Espécies em comum } & \multirow{2}{*}{ Fonte } \\
\hline & & & & $\%$ & valor absoluto & \\
\hline Campo/Mata de Araucária & Telêmaco Borba & & & 4 & 1 & Marinoni et al. 1999 \\
\hline Floresta subtropical & Ponta Grossa & 5 & 60 & 13 & 3 & Marinoni et al. 1999 \\
\hline Floresta subtropical & São José dos Pinhais & 5 & 60 & 38 & 9 & Marinoni et al. 1999 \\
\hline Floresta subtropical/Araucária & Guarapuava & 5 & 60 & 8 & 2 & Marinoni et al. 1999 \\
\hline Floresta tropical úmida & Costa Rica & - & - & 50 & 12 & Janzen, 1983 \\
\hline Floresta tropical úmida & Colombo & 5 & 60 & 21 & 5 & Marinoni et al. 1999 \\
\hline Floresta tropical úmida & Morretes & 1 & 12 & 46 & 11 & Laroca e Mielke 1975 \\
\hline Floresta tropical úmida & Jundiai do Sul & 5 & 60 & 33 & 8 & Marinoni et al. 1999 \\
\hline Floresta tropical úmida & Fênix & 5 & 60 & 13 & 3 & Marinoni et al. 1999 \\
\hline Floresta tropical úmida/Litoral & Antonina & 5 & 60 & 13 & 3 & Marinoni et al. 1999 \\
\hline Mata seca & Costa Rica & - & 130 & 58 & 14 & Haber \& Frankie 1989 \\
\hline
\end{tabular}

*) Cada coleta equivale a um esforço amostral de cerca de 12 horas. Os levantamentos foram realizados por um período médio de um ano

Com base na curva do número acumulado de espécies (Fig. 6), uma riqueza de espécies muito maior não é esperada para a região de estudo. A coleta em fontes luminosas representa um meio rápido e eficiente para se obter uma visão geral da composição em espécies da esfingofauna de um habitat ou de uma certa região. Entretanto, algumas espécies de esfingídeos não são atraídas pela luz (JANZEN 1983; KITCHING \& CADIOU 2000) e não devem ter sido registradas nesse levantamento.

Em ambientes sazonais, variações de parâmetros climáticos, como precipitação e temperatura, influenciam os ciclos de vida de plantas e de animais (e.g. OPLER et al. 1980; JANZEN 1983; HABER \& FRANKIE 1989). Na vegetação tipicamente sazonal do tabuleiro paraibano, meses com um maior número de espécies de plantas floridas devem, por conseqüência, apresentar uma maior riqueza de espécies dependente de recursos florais, como os esfingídeos. Contudo, no período de estudo foram registrados valores pluviométricos atípicos, de modo que estações seca e chuvosa não foram claramente definidas. Talvez por isso, a abundância e a riqueza dos esfingídeos não apresentaram correlações significativas com a precipitação registrada nesse período. Ainda assim, a maioria das espécies foi altamente sazonal, ocorrendo exclusivamente em meses secos ou chuvosos.

Oscilações da abundância e da riqueza dos esfingídeos devem ser determinadas por um conjunto de fatores, como presença de predadores, como morcegos (Chiroptera), disponibilidade de plantas esfingófilas e ciclos migratórios. Hipóteses para a causa de ciclos em populações de Lepidoptera têm invocado patógenos, parasitóides e interações presa-predador ou planta-herbívoro (BERRYMAN 1996). 


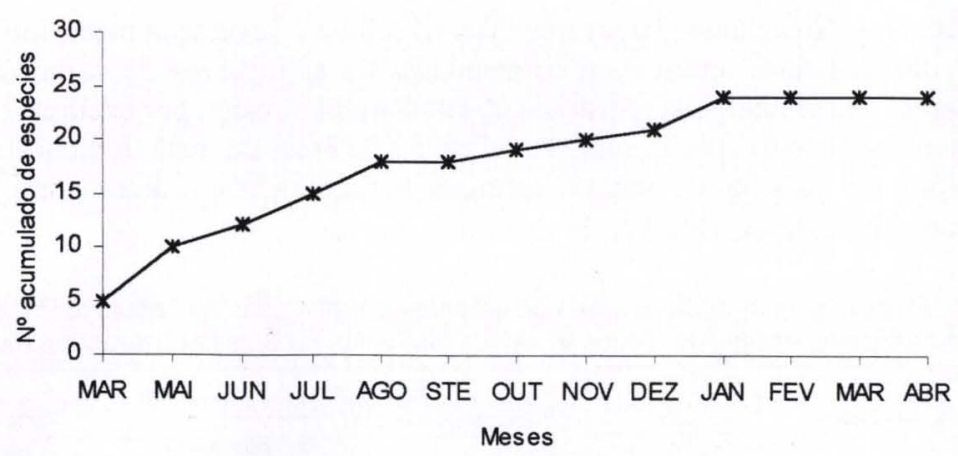

Fig. 6. Curva do número acumulado de espécies de esfingídeos coletados na Reserva Biológica Guaribas de março de 1999 a abril de 2000. Não houve coletas em abril de 1999.

A ausência de outros estudos da esfingofauna nordestina dificulta a determinação da ocorrência de padrões sazonais dos esfingídeos. Para tal, estudos futuros de comunidades esfingídeos-plantas esfingófilas devem ser plurianuais e considerar a história natural dos esfingídeos e a polinização das plantas que eles utilizam.

A influência dos parâmetros climáticos na atividade dos esfingídeos pode diferir de uma região para outra. Em diferentes localidades do Paraná, por exemplo, a temperatura parece ser um fator limitante da ocorrência de diversos grupos de insetos (MARINONI \& DUTRA 1991). No Tabuleiro Paraibano valores de temperaturas médias mensais flutuam de 24 a $26^{\circ} \mathrm{C}$. Neste ambiente, a ocorrência de temperaturas altas e constantes ao longo do ano faz desta variável climática um fator não limitante à ocorrência e à atividade dos esfingídeos.

\section{Relação com plantas esfingófilas}

Os esfingídeos apresentaram alta flexibilidade em relação aos hábitos e aos tipos florais das plantas visitadas. As espécies mais abundantes e registradas durante quase todo ano, Erinnyis ello e Isognathus menechus, visitaram flores de diferentes formas, incluindo as hipocrateriformes, em forma de disco, goela e pincel.

As espécies de plantas mais visitadas, Guettarda platipoda e Hancornia speciosa, são características do Tabuleiro Nordestino. Apresentam floração maciça e são tipicamente esfingófilas. Indivíduos de $H$. speciosa floresceram na estação seca e os de G. platipoda estiveram floridos nas estações seca e chuvosa, totalizando pelo menos oito meses de floração. Estas duas espécies devem apresentar um papel chave na comunidade esfingídeos-plantas do tabuleiro paraibano.

Algumas espécies de plantas visitadas pelos esfingídeos, como as de Capparis e de Inga, apresentam flores do tipo pincel. Estas espécies podem estar - subrepresentadas na análise polínica, pois ao serem visitadas por um esfingídeo, uma maior quantidade de pólen deve ser depositadas na cabeça do animal e não nas peças bucais. 
Das 14 plantas identificadas, 10 são tipicamente esfingófilas. Esfingídeos, contudo, também utilizaram flores melitófilas, como as de Cuphea flava, ao anoitecer. As flores de $C$. flava são ricas fontes de néctar para abelhas de diversas espécies.

Flores esfingófilas também foram visitadas por outros insetos. Flores de Hancornia speciosa, por exemplo, foram utilizadas, pela manhã, por abelhas (Euglossini) e borboletas (Hesperiidae e Nymphalidae).

Baseando-se no comprimento da espirotromba dos esfingídeos, parece haver pouca especificidade na escolha de flores. Espécimes com espirotromba curta os com espirotromba longa visitaram flores das mesmas espécies de plantas e com tipos florais semelhantes. Tanto indivíduos de Perigonia pallida e de Aellopos fadus, que apresentaram as espirotrombas mais curtas, quanto os de Agrius cingulatus e de Manduca paphus, com espirotrombas de comprimento maior que $8 \mathrm{~cm}$, visitaram flores de Guettarda platipoda as quais exibem tubo curto, Hancornia speciosa com tubo médio e de Luehea paniculata, as quais não apresentam tubo floral.

Ao nível de espécies de esfingídeos, não encontramos indicações de relações específicas com determinadas espécies de plantas. O mesmo foi verificado no único estudo de comunidade de esfingídeos e plantas esfingófilas realizado na região Neotropical, na Costa Rica (HABER \& FRANKIE 1989).

O tamanho da espirotromba dos esfingídeos, no entanto, deve influir na divisão do recurso alimentar, nos casos de flores com tubos florais muito compridos, como os de flores de Tocoyena, também ocorrente no Tabuleiro Paraibano. SIBERBAUER-GOTTSBERGER \& GOTTSBERGER (1975) registraram visitas de Agrius cingulatus a flores de Tocoyena brasiliensis. Contudo não foram encontrados grãos de pólen dessa planta na espirotromba dos esfingídeos.

Do ponto de vista das plantas esfingófilas, relações específicas com esfingídeos são esperadas para plantas com tubos florais extraordinariamente compridos. Nestes casos, o néctar produzido ou depositado no fundo do tubo floral, é disponível apenas para espécies de esfingídeos com espirotrombas extremamente longas, como Cocytius antaeus. Exemplos de relações entre esfingídeos e plantas altamente especializadas têm sido reportados em Madagascar. Tais casos envolvem relações de uma ou poucas espécies de esfingídeos como polinizadores de orquídeas com calcares gigantes de até $43 \mathrm{~cm}$ (NILSSON et al. 1985, 1992; NILSSON 1988, WASSERTHAL 1997).

Um terço dos esfingídeos apresentou carga polínica mista, incluindo pólen de mais de três espécies de plantas (Fig. 4). Esses esfingídeos devem apresentar uma estratégia de forrageamento oportunista. Ao utilizar várias espécies de plantas como fonte de alimento, visitantes florais podem diminuir sua eficiência como vetores de pólen entre plantas coespecíficas (WASER 1978). Dos demais esfingídeos com pólen nas espirotrombas, cerca de 70\%, apresentaram pólen de apenas uma a duas espécies de plantas. Como ocorre entre as abelhas oligoléticas, a fidelidade dos visitantes florais a uma ou poucas espécies de plantas aumenta sua eficiência como polinizadores (ROUBIK 1989; SCHLINDWEIN \& WITTMANN 1997). A utilização de um curto espectro de plantas e o transporte de grandes cargas polínicas nas peças bucais fazem dos esfingídeos os principais polinizadores das espécies noturnas do tabuleiro, como Hancornia speciosa e Guettarda platipoda. 


\begin{abstract}
AGRADECIMENTOS. Somos gratos ao Marcelo Marcelino e toda equipe da Reserva Biológica Guaribas (IBAMA): Sr. Sadan, Sr. Manoel Pedro, Sr. Oscar, Sr. Luís e Sr. Tabosa, pelo apoio logístico. Ao Dr. Olaf Mielke (UFPR) pelo auxílio na identificação da maior parte dos esfingídeos coletados. Ao Dr. Hans-Peter Stika e à Dra. Anne Heller (Universidade de StuttgartHohenheim) pela ajuda no uso do microscópio eletrônico. Ao Dr. Celso F. Martins (UFPB) e ao Sr. Jorge Kesselring pelo apoio. Ao Dr. Ricardo Rosa pela revisão do Abstract. À Cristiane F. Costa, Antônio J.C. Aguiar, Lemuel O. Leite e Neila Leite pela ajuda em campo. À CAPES, CNPq e Fundação A.F.W. Schimper pelo apoio financeiro.
\end{abstract}

\title{
REFERÊNCIAS BIBLIOGRÁFICAS
}

Andrade-Lima, D.1960. Estudos fitogeográficos de Pernambuco. Arq. Inst. Pesq. Agron. Pernambuco 5: 305-341.

BawA, K.S. 1990. Plant pollination interactions in tropical rain forests. Annu. Rev. Ecol. Syst. 21: 399-422.

BERRYMAN, A.A. 1996. What causes population cycles of forest Lepidoptera? TREE 11 (1): 28-32.

Biezanko, C.M. 1948. Sphingidae de Pelotas e seus arredores. Contribuição ao conhecimento da fisiografia do Rio Grande do Sul. Pelotas, Edição do Autor, 8p.

D'Abrera, B. 1986. Sphingidae Mundi. Hawk moths of the world. Faringdon, UK E. W. Classey Ltd., 226p.

FAEGRI, K \& L. VAN DER PIJ. 1979. The principles of pollination ecology. Oxford, Pergamon Press, 291p.

FERREIRA, P.S.F.; D.S. MARTINS \& N. HÜBNER. 1986. Levantamento flutuação e análise entomofaunística em mata remanescente da Zona da Mata, Viçosa, Minas Gerais. I. Sphingidae: Lepidoptera. Rev. Ceres, Viçosa, 33: 516-527.

FrANKIE, G.W.; P.A. OPLER \& H.G. BAKER. 1974. Comparative phenological studies of trees in tropical wet and dry forests in the lowlands of Costa Rica. Jour. Ecol. 62: 881-919.

HABER, W.A. \& G.W. FrANKIE. 1982. Pollination of Luehea (Tiliaceae) in Costa Rica deciduous forest. Ecology 63: 1740-1750.

. 1989. A tropical hawk-moth community: Costa Rican Dry Forest Sphingidae. Biotropica 21: $155-172$.

IвAMA.1995. Plano de ação emergencial: Reserva Biológica Guaribas. Ministério do Meio Ambiente dos Recursos Hídricos e da Amazônia Legal. Brasília, IBAMA, 66p.

JANZEN, D.H. 1967. Syncronization of sexual reproduction of trees with the dry season in Central America. Evolution 21: 620-637.

1983. Costa Rica natural history. Chicago, Univ. Chicago Press, 816p.

KITCHING, I.J \& J.M CADIOU. 2000. Hawkmoths of the world: An annotated and illustrated revisionary checklist (Lepidoptera: Sphingidae). London, Cornell Univ. Press, 226p.

LAROCA, S. \& O.H.H. MieLKE. 1975. Ensaios sobre a ecologia de comunidade em Sphingidae na Serra do Mar, Paraná, Brasil (Lepidoptera). Rev. Brasil. Biol. 35 (1): 1-18.

LAROCA, S.; V.O. BECKER \& F.C.V.ZANELLA. 1989 [1990]. Diversidade, abundância relativa e fenologia de Sphingidae (Lepidoptera) na Serra do Mar (Quatro Barras, PR), sul do Brasil. Acta Biol. Paranaense, Curitiba, 18: 13-53.

LoCATELl, E. \& I.C.S. MACHADO. 1999. Floral Biology of Cereus fernambucensis: a sphingophilous cactus of restinga. Bradleya, York, 17: 86-94.

Louveaux, J; A. Maurizio \& G. Vorwohl. 1978. Methods of melissopalynology. Bee World 59 (4): 139-157.

MARINONI, R.C. \& R.R.C. Dutra. 1991. Levantamento da fauna entomológica no estado do Paraná. Introdução. Situações climática e florística de oito pontos de coleta. Dados faunísticos de agosto de 1986 a julho de 1987. Revta bras. Zool. 8 (1/2/3/4): 31-73. 
Marinoni, R.C.; R.R.C. Dutra \& O.H.H. Mielke. 1999. Levantamento da fauna entomológica no Estado do Paraná. IV. Sphingidae (Lepidoptera). Diversidade alfa e estrutura de comunidade. Revta bras. Zool. 16 (Supl. 2): 223-240.

Nilsson, L.A. 1988. The evolution of flowers with deep corolla tubes. Nature 334 (6177): 147-149.

NilsSON, L.A., E. RabakonANDRIANINA \& B.PETTERSSON. 1992. Exact tracking of pollen transfer and mating in plants. Nature 360: 666-667.

NILSSON, L.A; L. JONSSON \& E.R. RANDRIANJOHANY. 1985. Monophily and pollination mechanisms in Angraecum arachnites Schltr. (Orchidaceae) in a guild of long-tongued hawk-moths (Sphingidae) in Madagascar. Biol. Jour. Linn. Soc. Lond. 26: 1-19.

Oliveira-Filho, A.T. \& D.A. DE CARvalho. 1993. Florísitca e fisionomia da vegetação no extremo norte do litoral da Paraíba. Rev. Brasil. Bot. 16 (1): 115-130.

Oliveira-Filho, A.T.; G.J. Shepherd; F.R. Martins \& W.H. Stubblebine. 1986. Enviromental factors affecting physiognomic and florisitc variation in a area of cerrado in central Brazil. Jour. Trop. Ecol. 5: 413-431.

OPLER, P.A.; G.W. Frankie \& H.G. BAKER. 1980. Comparative phenological studies of treelet and shrub species in tropical wet and dry forests in the lowlands of Costa Rica. Jour. Ecol. 68: 167-188.

Prates, M.; L.C.S. Gatto \& M.I.P. Costa. 1981. Geomorfologia - Projeto RADAMBRASIL, Levantamento de Recursos Naturais. Rio de Janeiro, Ministério das Minas e Energia, Vol. 23, p. 301-348.

RoubIK, D.W. 1989. Ecology and natural history of tropical bees. Cambridge Univ. Press, 514p.

SCHLindwein, C. \& D. WitTmann. 1997. Stamen movements in flowers of Opuntia (Cactaceae) favour oligolectic bee pollinators. Plant Syst. Evol. 204: 179-193.

Silberbauer-GotTSBerger, I. \& G. GotTSBERGER. 1975. Über sphingophile Angisopermen Brasiliens. Plant Syst. Evol. 123:157-184.

STEIFERT, R.P. 1974. The Sphingidae of Turrialba, Costa Rica. Jour. N.Y. Ent. Soc. 82: 45-56

STEINER, A. \& I. NikUSH. 1994. Beobachtungsmethoden bei Nachtfaltern, p. 28-50. In: G. EBERT (Ed.). Die Schmetterlinge Baden-Württembergs. Band 3 Nachtfalter I. Stuttgart, Ulmer, 518p.

TAVARES, R. 1988a. Inventário da vegetação dos tabuleiros do Nordeste. Coleção Mossoroense, série B, Mossoró, 493: 11-12.

-1988b. Contribuição para o estudo da cobertura vegetal dos tabuleiros do Nordeste. Coleção Mossoroense, série B, Mossoró, 494: 13-25.

Vogel, S. 1954. Blütenbiologische Typen als Elemente der Sippengliederung Botanische Studien. Jena, Gustav Fischer Verlag, 339p.

WASER, N.M. 1978. Interspecific pollen transfer and competition between co-occuring plant species. Oecologia 36: 223-236.

WASSERTHAL, L.T. 1997. The pollinators fo the malagasy star orchids Angraecum sesquipedale, A. sororrium and A. compactum and the evolution of extremely long spurs by pollinator shift. Bot. Acta, Stuttgart , 110: 343-359.

WittMann, D. \& C. SChlindwein. 1995. Melittophilous plants, their pollen and flower visiting bees in Southern Brazil 1: Loasaceae. Biociências, Porto Alegre, 3: 19-34.

WolDA, H. 1978. Fluctuations in abundance of tropical insects. Amer. Nat. 112: 1017-1045.

Recebido em 08.VI.2001; aceito em 07.V.2002. 